\title{
General quality planning in the hemostasis laboratory
}

\author{
J.D. Olson
}

"So", you might ask, "What is quality, anyway?" The word quality repeatedly infiltrates our discussions and interactions as we work to produce or choose a product. The Oxford English Dictionary devotes more that 3000 words in its effort to define the many variations on the use of this word. We may all have difficulty with a definition, but we do know what we mean. The customer of the product or service defines many aspects of its quality while those who are producing define many others. Stated in its simplest terms, quality is the condition or state of a person, thing or process.

Elements of quality management systems began with a publication by Shewhart in $1931^{1}$ providing a footing for quality processes based on a scientific and/or statistical footing. He stated: “A phenomenon will be said to be controlled when, through the use of past experience, we can predict, at least within limits, how the phenomenon may be expected to vary in the future. Here it is understood that prediction means that we can state, at least approximately, the probability that the observed phenomenon will fall within given limits." 1

The evolution of quality management systems were influenced by experiences in World War II. During the war, individuals involved in the production of reliable devices (weapons and other implements to support a war effort) for the consumer (soldier) to do their job effectively tied the entire system from raw

Quality in Laboratory Hemostasis and Thrombosis, 1st edition. By Steve Kitchen, John D. Olson and F. Eric Preston. Published 2009 by Blackwell Publishing. ISBN: 978-1-4051-6803-8 material to the use of the finished product in a unique "team" from start to finish. Few circumstances can link the person in production so directly to the importance of the outcome. The success of the soldier was tied to the long-term well-being of the person making the weapon or other defense device. This ability to build the tight feeling of kinship and team on the part of people in production to the quality of the product is the goal of quality programs in all sectors of the economy today. It is, of course, very difficult to achieve this attitude in the workplace in the same way that it could be when the outcome could so directly benefit the producers.

Following World War II, the effort of reconstruction of the industry and economy of the affected countries became a major international effort and influenced the evolution of quality programs. The work of Deming ${ }^{2}$ and Juran, ${ }^{3,4}$ both associates of Shewhart, extended his work. In 1951, Juran ${ }^{3}$ published a seminal book that proposed the key elements for managing quality: quality planning, quality control and quality improvement. Following World War II, Deming proposed a significant departure from the "standard" thinking about quality, proposing that modification to the real relationships of quality, costs, productivity and profit. The different approach to quality espoused by Deming is compared with the "standard" thinking in Table 1.1.5 Thus, anything that improves the product or service in the eyes of the customer defines the goals of the quality program.

Organizations that follow Deming principles find that good quality is hard to define, but the lack of quality is easily identified. In the "standard" management of a system, the workers ultimately pay for 
Table 1.1 Comparison of Deming and traditional management principles. $^{5}$

\section{Common company practices}

- Quality is expensive

- Inspection is the key to quality

- Quality control experts and inspectors can ensure quality

- Defects are caused by workers

- The manufacturing process can be optimized by outside experts with little or no change in system afterwards

- Little or no input from workers

- Use of work standards, quotas and goals can help productivity

- Fear and reward are proper ways to motivate

- Employees can be treated like commodities - buying more when needed, laying-off when needing less

- Rewarding the best performers and punishing the worst will lead to greater productivity and creativity

- Buy one supplier off against another and switch suppliers based only on price

- Profits are made by keeping revenue high and costs down

“Deming” company practices

- Quality leads to lower costs

- Inspection is too late. If workers can produce defect-free goods, eliminate inspections

- Quality is made in the boardroom

- Most defects are caused by the system

- Process is never optimized; it can always be improved

- Elimination of all work standards and quotas is necessary. Fear leads to disaster

- People should be made to feel secure in their jobs

- Most variation is caused by the system

- Buy from vendors committed to quality and work with suppliers

- Invest time and knowledge to help suppliers improve quality and costs. Develop long-term relationships with suppliers

- Profits are generated by loyal customers

management failure; when profits are reduced, then management reduces labor costs. In contrast, moving quality programs as close to the worker as possible will ultimately lead to lower cost and improved consumer and worker satisfaction.
The clinical laboratory has three "consumers" of their product:

1 The patient who benefits from the best possible result.

2 The ordering clinician who depends upon the right test, at the right time, with an accurate result in order to make a clinical decision.

3 The hospital, clinic or other entity that depends upon the laboratory for a positive margin when comparing cost with revenue.

All three consumers benefit when the quality program drives the best possible practice.

Many different quality practices and/or programs have evolved in the decades since the original work of Shewhart, Juran and Deming. They all have acronyms (e.g. TQM, CQI, ISO, IOP, ORYX, Six Sigma ${ }^{\circledR}$ ) and a common goal of improving the quality of the performance (and product) of an organization. The discussion of these individual programs is beyond the scope of this chapter, but many of the principles are addressed below and in other chapters of this book. All programs have great strength, but they also suffer from being proscriptive, an issue that is discussed later in this chapter.

\section{Elements of quality in the hemostasis laboratory}

At the moment that a clinician orders a laboratory test, he/she sets in motion a complex process that involves many individuals. More than two dozen individual actions, involvement of sophisticated instruments and multiple interfaces of computing devices encompass three phases: preanalytic phase (order, collection and transport); analytic phase (making the correct measurement); postanalytic phase (formulating and delivering the data and the action of the clinician in response to the result). Figure 1.1 is a graphic depiction of this process. Examining the figure, one might think of each arrow representing an opportunity for error that could effect the final result. A quality program must encompass all of these events with process to prevent errors and to detect an error if it occurs.

Internal quality control

The control of the testing procedure (quality control) evolved with the transition of research testing into the 


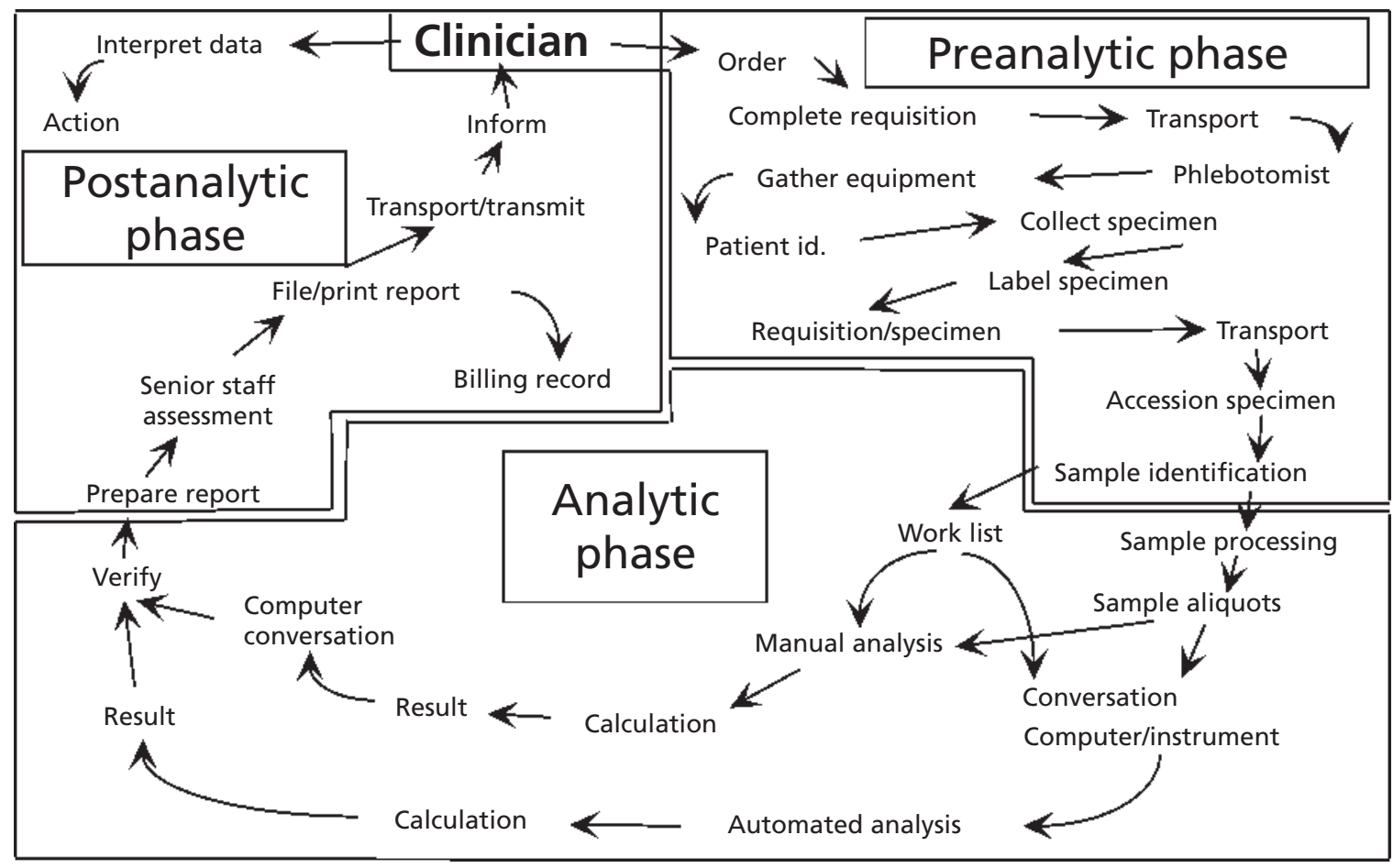

Fig. 1.1 The laboratory cycle. Depicted are the steps needed to complete a laboratory test, beginning with the ordering clinician and ending with the response of the ordering clinician to the result. Preanalytic, analytic and

clinical arena. To be confident that the method returns the correct result requires that steps be taken to assure all elements are within the control of the operator. Technologists are taught that instruments/methods are designed to fail and that they can rely upon results only if the entire method performs within defined limits with specimens of known value. The frequency of these control events are method specific and a function of the stability of all of the elements (reagent, specimen, instrument) and must be driven by historical data from the method itself. Internal quality control is the grandfather of quality programs in the laboratory and is detailed elsewhere in this book (Chapter 5).

Many industries and some laboratories have adopted control processes that focus on quantifying and reducing errors called Six Sigma ${ }^{\circledR}{ }^{6}$ Six Sigma ${ }^{\circledR}$ was developed by an engineer (Bill Smith) at the Motorola company and the company began using the program in the mid 1980s. Six Sigma ${ }^{\circledR}$ is a registered postanalytic parts of the process are indicated. More that two dozen steps, indicated by arrows, are involved, each of which may be the source of an error. Monitoring by a quality program is required.

trademark of the Motorola Corporation. Application of the process has become very popular among companies internationally. Six Sigma ${ }^{\circledR}$ processes can be applied to discrete events (e.g. mislabeled specimens, clerical errors) and to variable events (i.e. variance of a method like the fibrinogen assay). Elements of these activities are depicted in Table 1.2. Discrete elements are expressed in defects per million events (DPM). Achieving the Six Sigma ${ }^{\circledR}$ goal means that defects are less than $1: 1,000,000$, a level achieved in the airline industry. Errors in the healthcare industry are much more frequent, with errors causing injury to hospitalized patients at 10,000 DPM (3.8 $\sigma)$, errors in therapeutic drug monitoring 244,000 DPM $(2.2 \sigma)$ or errors of laboratory reporting much better at 447 DPM $(4.8 \sigma){ }^{7}$

Other aspects of the laboratory activity rely on analysis of the variability of data. This variability can be measured at several levels. The greatest variability is seen in External Quality Assessment (EQA) data 
Table 1.2 Six Sigma ${ }^{\circledR}$ metrics. $^{6}$

Measure outcomes

Inspect outcomes and count defects

Calculate defects per million

Convert DPM to sigma metric

\section{Measure variation}

Measure variation of a process (SD)

Calculate sigma process capability

Determine QC design metric

DPM, defects per million events; QC, quality control; SD, standard deviation.

Table 1.3 Sigma metrics for common coagulation tests. ${ }^{8}$

\begin{tabular}{lllll}
\hline \multirow{5}{*}{ Test } & \multicolumn{3}{l}{ Sigma metric } & \\
\cline { 2 - 5 } & TEa & NTQ & NMQ & LMQ \\
\hline Prothrombin time & $15 \%$ & na & 1.77 & 5.35 \\
INR & $20 \%$ & na & 2.39 & 3.52 \\
Fibrinogen assay & $20 \%$ & 1.78 & 2.01 & 3.24 \\
\hline
\end{tabular}

INR, international normalized ratio; LMQ, Local Method Quality (single laboratory); na, not available; NMQ, National Method Quality (within method); NTQ, National Total Quality (all methods); TEa, total acceptable error.

regarding the all method variance, referred to as the National Total Quality (NTQ). EQA programs also report data for an analyte comparing many laboratories using the same method, referred to the National Method Quality (NMQ). NMQ is frequently significantly better because variability is only among laboratories using the same methods, but not among methods. The lowest variability is seen with a single method in a single laboratory, referred to as the Local Method Quality (LMQ). ${ }^{8}$ Thus, the degree of variability is best controlled at the local level, methodspecific interlaboratory variability is greater variability and the greatest is the variability examining across all methods. Examples of this degree of variability are shown for prothrombin time, international normalized ratio (INR) and fibrinogen assay in Table $1.3 .^{8}$ The data in Table 1.3 are very specifically based on the data from the 2004 EQA data of the College of
American Pathologists, as reported by Westgard. ${ }^{8}$ Should a number of different EQA data sets be analyzed, there would be a range of sigma statistics of a similar magnitude. The low sigma values shown mean that adequate control will demand more rigorous attention to control procedures, often necessitating multiple control rules. Common goals in industry are to strive for $6 \sigma$ processes and to accept $3 \sigma$. At $3 \sigma$ or below, effective error detection could not be achieved, even with as many as six quality control rules. There is much progress yet to be made in the quality of many coagulation procedures.

\section{Quality Assurance}

During the 1980s, laboratories began looking beyond the analytic procedure with quality programs called Quality Assurance. Quality control remained a part of the Quality Assurance program, but the program expanded to consider such items as laboratory orders, requisitions, collections techniques and other issues directly impacting the result of the test but not always directly in the control of the laboratory. Preanalytic issues are detailed elsewhere in this book (Chapter 4). Postanalytic issues also became a part of quality initiatives this same era. Such issues as reporting formats, verification of calculated results, timely reporting and even action taken as a result of the data reported. It was during this period that computer applications in both the laboratory and the clinical environments began to grow, requiring the validations and continued verification of computer function and interfaces for electronic result reporting between computers as well as between instruments and computers. Encouraged (or demanded) by accreditation and/or regulatory agencies, laboratory professionals also began asking questions of and listening to clinicians regarding the quality of service, needs to provide new tests shown to have clinical value and to remove antiquated tests that no longer offer added clinical information. These activities started the interaction of the quality programs in the laboratory with similar programs in the rest of the healthcare institution.

\section{External Quality Assessment}

In the $1930 \mathrm{~s},{ }^{9}$ the need for interlaboratory standardization for public health programs led to early efforts at EQA. The concept of an unknown specimen being 
sent from a central EQA agency to the laboratory for testing with the results sent back to the agency for evaluation added an important new level of assurance for the quality of analysis. In addition, results were reported in a way that allowed a laboratory to compare their performance with other laboratories using the same or similar methods. Laboratory participation in EQA programs grew rapidly in the 1950s and 1960s. In large part this growth was due to the development of accreditation and regulatory programs requiring EQA; however, the recognition by unregulated laboratories that EQA was vital to the quality of their own programs has also led to widening acceptance. Detailed discussion of EQA programs is addressed elsewhere in this book (Chapter 6).

\section{Error detection and correction}

McGregor ${ }^{10}$ contrasted two theories of company management which he referred to as $\mathrm{X}$ and $\mathrm{Y}$. A company following theory $\mathrm{X}$ assumes that the worker prefers to be directed and wants to avoid responsibility. In contrast, a company that is following theory $\mathrm{Y}$ assumes that the workers enjoy what they do and, in the right conditions, will strive to do their very best. In general, the company that follows theory $\mathrm{X}$ manages from the "top down" with dependence of the worker upon management as he/she performs tasks. A hallmark of theory $\mathrm{X}$ is toughness, the rules are laid out and every employee must "obey." The workplace has an element of fear that an error might occur and that reprimand will result. The style of the company that follows theory $\mathrm{Y}$ is different. Management works from the "bottom up." The workplace is configured to satisfy the worker and to encourage commitment to the organization. Workers are encouraged to be self-directed and the management and supervisory style is supportive. Theory $\mathrm{Y}$ has been described as operating with a "velvet glove." Stated in another way, one can think of management under theory X strives to "drive" the organization and the workers to success, while the management under theory Y strives to "lead" the organization and the workers to success. The goal in both cases is essentially the same, but the means are very different. This brief description of diverging management styles can impact process improvement within the laboratory.

In order for any method, process or laboratory to improve, it is paramount to correct and understand the cause of the errors that interfere with productivity. The laboratory needs a system for capturing and categorizing errors. Such a system becomes the infrastructure for improvement in a quality program. It is obvious that for such a system to be successful, there needs to be an aggressive program to identify all errors, optimally at the time of the occurrence and the ideal process is one that is looking prospectively at processes, seeking to prevent. Deming ${ }^{5}$ pointed out that inspection after the fact is too late. The airline industry provides an example. Considerable effort is applied to understanding what causes the big error, an airplane crash. However, great efforts are now directed at the near misses both in the air and on the ground, a proactive effort to understand the "close call" to help prevent the major event. The laboratory needs a similar aggressive approach that must begin with each individual owning their part of the process and identifying the problems as they occur, or seeing ways to prevent problems by changing process. In order for such a process to be most efficient, the worker cannot be threatened by the mechanism to report errors. The examples regarding the differing approaches may be useful.

First, a technologist has just completed a run on an automated instrument using expensive reagents and many patient results. He/she notices that two required reagents were placed in the wrong position, causing them to be added in the wrong order. The error caused erroneous patient results, but not to the degree that it would be easily detected. The consequence of repeating the run is twofold: the cost of the reagent and time of the technologist are expensive and the delay in completing the testing resulting in complaints from clinicians. In this scenario, management under theory $\mathrm{X}$ results in a reprimand from the supervisor and a letter being placed in the technologist's personnel file for consideration at the next performance evaluation. The consequences may be severe enough for the technologist to consider not reporting the error. In contrast, management under theory Y would result in the supervisor complementing the technologist for detecting the problem and engaging the technologist in an investigation of the reason that the error occurred. The supervisor and the technologist understand that the goal is to prevent this from happening in the future, whether this person or another performs the procedure. 
Second is a case in which the error that occurred above was not detected by the technologist performing the test, but at a later time during the supervisor's inspection of reported results. Managing under theory $\mathrm{X}$, the supervisor will confront the technologist with the data and, just as in the prior example, will issue a reprimand and a letter. Managing under theory Y, the supervisor will present the information to the technologist and ask the technologist to assist in understanding how the problem occurred and how it might be avoided in the future.

Errors like those described that are detected and investigated are most frequently found to be problems in the process, not exclusively with the individual carrying out the procedure at the time. Improving the process to help workers prevent errors is the goal, which can only be achieved if all errors are detected and investigated. Contrasting the approaches, one can see that punishing the worker and failing to examine process will not improve the quality and the worker will not be enthused about reporting future errors. The second approach engages the workers and rewards activities that improve quality in the laboratory.

\section{Quality System Essentials}

Development and maintenance of a quality program in a laboratory requires that there be an infrastructure of support in order for internal and external quality control and quality assurance to be successful. The field of hemostasis provides an excellent example of this issue. The hemostasis laboratory has the entire spectrum of testing from the highly automated to the complex manual tests that are time-consuming and demand a different skill set. Thus, in addition to a good quality control program, there is need for an effective program for development and continuing education of the staff. The same can be said of a host of essential activities in the laboratory including such things as: acquisition and maintenance of capital equipment; supply inventory; safety of staff and patients; and others. In the late 1990s and early 2000s, recommendations began to appear for the comprehensive management of the quality of all aspects of the laboratory operations. International Standards Organization (ISO) developed the ISO 17025 (primarily a laboratory management program) ${ }^{11}$ and ISO 15189 (a program specifically for clinical
Table 1.4 Quality System Essentials (CLSI 1999). ${ }^{11}$

Purchasing and inventory

Organization

Personnel

Equipment

Document and records

Process control

Information management

Deviations, non-conformance

Assessments: internal and external

Process improvement

Customer service

Facilities and safety

laboratories). ${ }^{12}$ The Clinical Laboratory Standards Institute (CLSI), at the time named NCCLS, published the Quality System Essentials (QSE). ${ }^{13}$ The ISO programs have achieved acceptance in Europe, while the QSE programs are more commonly in use in North America. Both approach the issues of quality with a very broad perspective, covering all elements of laboratory operations. There is a brief description of some aspects of QSE below.

The list presented in Table 1.4 is an example of the QSE for a given laboratory. The list is not intended to be the list for use in every laboratory. Each laboratory needs to develop its own essentials, formulated to help manage issues within their own laboratory. The list is ordinarily 9-12 items in length and the types of issues to be addressed are encompassed in Table 1.4. Each of the item on this list will be controlled by a set of three levels of documents:

\section{Policies}

Statement of intent with regards to rules and requirements of regulations, accreditation and standards. Each QSE will have one or a small number of policies that will provide the framework for all activities within the QSE. In the case of test development, policies may address such things as validation, quality control, EQA and others.

\section{Process descriptions}

This is a description of how the policies are implemented. Process descriptions will often cross more than one department, section of departments and procedures within a department. Flowcharts and tables 
Fig. 1.2 Process for the bleeding profile. This process for reporting the results of the prothrombin time (PT), fibrinogen assay (fib assay), platelet count (Plt Ct) and hemoglobin ( $\mathrm{Hgb})$ involves the activity of at least four different units in the health system and execution of as many as 10 standard operating procedures (SOPs). As a part of the Quality System Essentials (QSE), a process description would be needed to assure return of results rapidly enough for clinician action when managing an actively bleeding patient.

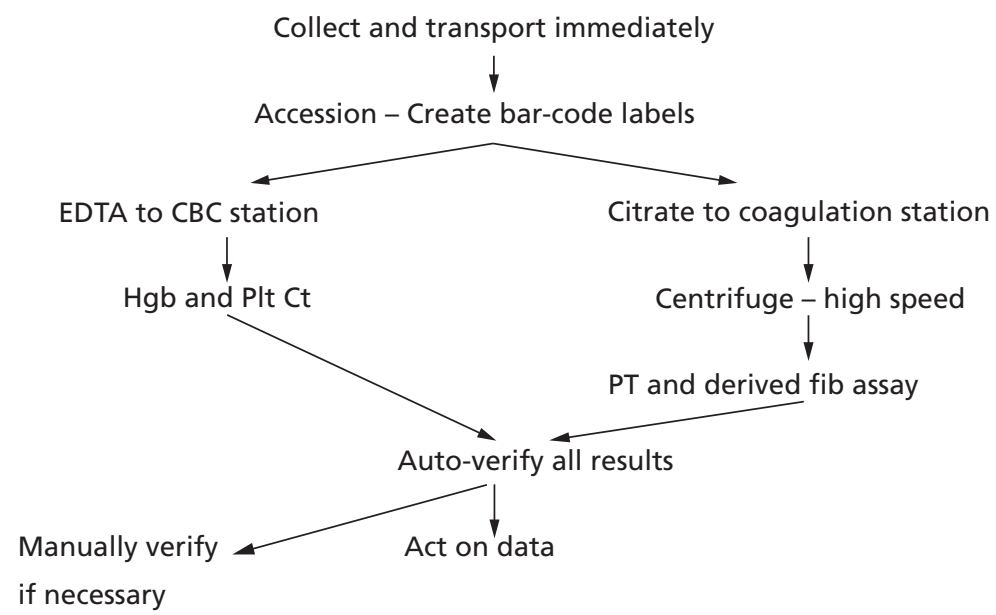

are often used to describe processes. An example of a process requiring control is given below.

\section{Procedures and related forms}

The standard operating procedure (SOP) is a step-bystep description of how to perform an individual method or task.

The policy and the SOP are commonly used documents in all laboratories; however, the process description may not be as familiar. An example is shown in Fig. 1.2. The purpose of this process is to provide the surgeon and anesthesiologist with information needed to manage blood transfusion therapy in the rapidly bleeding patient. The data needed are the prothrombin time, fibrinogen, hemoglobin and platelet count. The process needs for an order, specimen collection, transport, laboratory receipt/accession, testing in two separate sections of the laboratory, reporting and delivery of the data to the clinician. Ownership of the various steps in this process are in the control of the physician, nurse and three different sections of the laboratory. In order for this to occur in a meaningful time frame in the clinical setting (less than 15 minutes), there must be well-understood coordination among all of those involved. Each step in the process described has its own SOP for the action taken. In this case, there are at least 10 SOPs supporting a single process.

Implementation of a program can be challenging. Most laboratories have a quality program that can provide the beginning for the development of QSE.
Most laboratories also have most of the essentials that they will define in their QSE, they are just not under the umbrella of the program and not easily identified. Thus, an initial step in changing the program will be gathering key individuals with knowledge and energy for the process to identify the QSE for the organization. Technologists should also be represented in this process. Once the QSE are identified, teams can be formed to begin drafting of policies. Leadership from the highest levels, supporting the changes that need to be made and leading the infrastructure of a management structure based upon McGregor's theory Y is a crucial element. Possibly the most important issue is putting reality into faultfree reporting of errors, followed by an investigation to improve process to prevent future occurrences.

For many laboratories, instituting the concepts that are described in this chapter would necessitate significant change in the quality program, the perspective of the manager and the attitude of the employee. Such a change in the culture is difficult. It is tempting to try to "buy, install and run" a program from a quality vendor. Such an approach is likely to meet with resistance, with workers viewing it as "just another of those quality things that the administration is going to force on us." In the past two decades (or more) most laboratories have instituted more than one new quality program in an effort to find a solution that works well in their setting. One possible difficulty in such an approach is the proscriptive nature of the process. They provide everything that is needed: 
policies, forms, SOPs, etc. What they do not provide is the personal ownership that can come from the internal development of the quality process. Managers may find a smoother and more lasting solution in providing policies that allow for each unit to develop their own approach to the gathering of data, the identification of errors and the many other elements of the quality program.

\section{Conclusions}

Over the course of the past 70 years or more, elements of the quality program have evolved in a somewhat stepwise fashion, beginning with internal quality control and progressing to more comprehensive programs that encompass all activities in the workplace. In the remainder of this book you will find information regarding quality in all aspects of the hemostasis laboratory. Experts provide information regarding the highest level of development of standards (both methods and materials) to the finest details of the nuances of selected methods. Integrated into a comprehensive quality program, similar to that described above, the information should help in the development of a "quality hemostasis laboratory."

\section{References}

1 Shewhart WA. Economic Control of Quality of Manufactured Product. New York: D. Van Nostrand, 1931.

2 DeAguayo R. Deming: The American Who Taught the Japanese About Quality. New York: Simon and Schuster, 1990: 1-66.
3 Juran JM. Juran's Quality Control Handbook, 4th edn. New York: McGraw-Hill, 1988.

4 Juran JM. Juran on Leadership for Quality: An Executive Handbook. New York: Collier Macmillan, 1989: $1-80$.

5 Travers EM, McClatchy KM. Basic laboratory management. In Clinical Laboratory Medicine, 2nd edn. McClatchy KM (ed.). Philadelphia: Lippincott, Williams and Wilkins, 2002: 3-31.

6 Westgard JO. Six Sigmas Quality Design and Control. Madison, WI: Westgard QC, 2001: 11-22.

7 Nevalainen D, Berte L, Kraft C, Leigh E, Morgan T. Evaluating laboratory performance on quality indicators with the Six Sigma scale. Arch Pathol Lab Med 2000; 124: 516-519.

8 Westgard JO, Westgard SA. The quality of laboratory testing today: an assessment of $\sigma$ metrics for analytic quality using performance data from proficiency testing surveys and the CLIA criteria for acceptable performance. Am J Clin Pathol 2006; 125: 343-354.

9 Cumming HS, Hazen HH, Sanford FE, et al. Evaluation of serodiagnostic tests for syphilis in the United States: report of results. Vener Dis Inf 1935, 16: 189.

10 McGregor D. The Human Side of Enterprise. New York: McGraw-Hill, 1960: 1-58.

11 International Organization for Standardization. ISO/IEC 17025:2005: General requirements for the competence of testing and calibration laboratories. http://www.iso. org/iso/catalogue_detail?csnumber $=39883$

12 International Organization for Standardization. ISO 15189. 2007: Medical Laboratories: Particular requirements for quality and competence. http://www.iso.org/ iso/iso_catalogue/catalogue_tc/catalogue_detail. htm? csnumber $=42641$

13 National Committee for Clinical Laboratory Standards. A Quality System Model for Health Care: Approved Guidelines. NCCLS Document GP26-A. Wayne, PA: NCCLS, 1999. 